Original Research Paper

\title{
A Certain Subclass of Meromorphically Multivalent Analytic Functions with Negative Coefficients
}

\author{
${ }^{1}$ Ahmad, M.Z., ${ }^{1,2}$ Hiba F. Al-Janaby and ${ }^{3}$ Rabha W. Ibrahim \\ ${ }^{1}$ Institute of Engineering Mathematics, Universiti Malaysia Perlis, 02600 Arau Perlis, Malaysia \\ ${ }^{2}$ Department of Mathematics, College of Science, University of Baghdad, Baghdad-Iraq \\ ${ }^{3}$ Faculty of Computer Science and IT, University Malaya, 50603 Kuala Lumpur, Malaysia
}

Article history

Received: 04-05-2015

Revised: $29-07-2015$

Accepted: 28-09-2015

Corresponding Author:

Rabha W. Ibrahim

Faculty of Computer Science

and IT, University Malaya,

50603 Kuala Lumpur, Malaysia

Email: rabhaibrahim@yahoo.com

\begin{abstract}
The paper aims to introduce a certain subclass $\mathrm{S}_{\mathrm{g}}{ }_{\mathrm{g}}(A, B, \alpha, p, j)$ of the class $\Sigma^{*}(p)$ of meromorphically multivalent functions with negative coefficients, defined by using the definitions of Hadamard product and subordination for two functions belong to the class $\Sigma^{*}(p)$. We first investigate the geometric characterization property, giving the coefficient estimates for functions in the class $\mathrm{S}_{\mathrm{g}}{ }_{\mathrm{g}}(A, B, \alpha, p, j)$. We also obtain the distortion theorem, radii of meromorphically $\mathrm{p}$-valent starlikeness and convexity of order $(0 \leq \gamma<p)$, neighborhood property, partial sums, convolution properties as well as integral operator and integral representation.
\end{abstract}

Keywords: Meromorphic Function, Multivalent Function, Subordination, Hadamard Product

\section{Introduction}

Let $\Sigma(p)$ denote the class of functions $f(z)$ of the form:

$$
f(z)=z^{-p}+\sum_{n=p}^{\infty} a_{n} z^{n}(p \in \mathbb{N}:=\{1,2,3 \ldots\})
$$

Which are analytic and p-valent (multivalent) in the punctured unit disk $U^{*}:=U^{*}(1)$ where.

$$
U^{*}(r)=\{z: 0<|z|<r(0<r \leq 1)\}=U(r) \backslash\{0\}
$$

Let $\Sigma^{*}(p)$ denote the subclass of $\Sigma(p)$ consisting of functions of the form:

$$
f(z)=z^{-p}-\sum_{n=p}^{\infty} a_{n} z^{n}\left(a_{n} \geq 0 ; p \in \mathbb{N}\right)
$$

Which are analytic and p-valent (multivalent) in the punctured unit disk $U^{*}$.

A function $f \in \Sigma^{*}(p)$ is said to be meromorphically pvalent starlike of order $(0 \leq \gamma<p)$ in $U^{*}(r)$ if $\operatorname{Re}\left\{-\frac{z f^{\prime}(z)}{f(z)}\right\}>\gamma,\left(z \in U^{*}(r) ; 0<r \leq 1 ; 0 \leq \gamma<p\right), \quad$ also a function $f \in \Sigma^{*}(p)$ is said to be meromorphically $p$-valent

$$
\begin{aligned}
& \text { convex of order }(0 \leq \gamma<p) \quad \text { in } U^{*}(r) \quad \text { if } \\
& \operatorname{Re}\left\{-\left(1+\frac{z f^{\prime}(z)}{f(z)}\right)\right\}>\gamma\left(z \in U^{*}(r) ; 0<r \leq 1 ; 0 \leq \gamma<p\right) \quad \text { (cf. }
\end{aligned}
$$

e.g., Duren, 1983; Goodman, 1983; Srivastava and Owa, $1992)$. Let $B$ be a subclass of the class $\Sigma^{*}(p)$. We define the radius of meromorphically $p$-valent starlike of order $\gamma$ and the radius of meromorphically $p$-valent convex of order $\gamma$ for the class $B$ by:

$$
\begin{aligned}
& \mathcal{R}_{\gamma}^{*}=\inf _{f \in \mathcal{B}}(\operatorname{supsup}\{r \in(0,1]: \\
& \left.f i s m e r o m o r p h i c a l l y p-\text { valent starlike of order } \gamma \operatorname{in} U^{*}(r)\right\}
\end{aligned}
$$

$$
\begin{aligned}
& \mathcal{R}_{\gamma}^{c}=\inf _{f \in \mathcal{B}}(\operatorname{supsup}\{r \in(0,1]: f \text { ismeromorphically } \\
& \left.p-\text { valent convex of order } \gamma \operatorname{in} U^{*}(r)\right\},
\end{aligned}
$$

The convolution or the Hadamard product of two meromorphic $p$-valent functions $f$ and $g$, where $f$ is given by (1.1) and $g(z)=z^{-p}-\sum_{n=p}^{\infty} b_{n} z^{n}\left(b_{n} \geq 0 ; p \in \mathbb{N}\right)$ is denoted by $f^{*} g$ and defined by (Aouf, 2009):

$$
\left(f^{*} g\right)(z)=z^{-p}-\sum_{n=p}^{\infty} a_{n} b_{n} z^{n}
$$


Recall (Aouf and El-Ashwah, 2009) that an analytic function $f$ is said to be subordinate to an analytic function $g$ written $f<g$, if $f(z)=g(w(z)),|z|<1$ for some analytic function $w$ with $|w(z)|<1$.

The significance of the geometric properties and characteristics of various interesting subclasses of the class $\Sigma(p)$ of meromorphically univalent and multivalent functions with negative coefficients or positive coefficients has comprehensively been investigated by many well-known complex analysts. For instance, (Uralegaddi and Ganigi 1987; Srivastava et al., 1998; Aouf and Hossen, 1993; Liu and Srivastava, 2004; Aouf and Shammaky, 2005; Aouf, 2009; Aouf and ElAshwah, 2009; Aouf, 2010; Kamali et al., 2011; Makinde, 2012).

By making use of the following definition for Hadamard product and subordination, a new subclass $\mathcal{S}_{g}^{*}(A, B, \alpha, p, j)$ of functions in $\Sigma^{*}(p)$ is introduced.

Definition 1.1. A function $f(z) \in \Sigma^{*}(p)$ is said to be in the class $\mathcal{S}_{g}^{*}(A, B, \alpha, p, j)$ if it satisfies the following subordination condition:

$$
\begin{aligned}
& \frac{z\left(\left(f^{*} g\right)(z)\right)^{(j+1)}}{\left(\left(f^{*} g\right)(z)\right)^{(j)}} \prec \\
& -\left[\frac{(p+j)+[B(p+j)-(p+j-\alpha)(A-B)] z}{1+B z}\right]
\end{aligned}
$$

where, $\quad-1 \leq \mathrm{A}<\mathrm{B} \leq 1 ; \quad 0<\mathrm{B} \leq 1 ; \quad 0 \leq \alpha<\mathrm{p}+\mathrm{j} ; \quad \mathrm{j} \leq \mathrm{p} \leq \mathrm{n}, \quad \mathrm{p} \in \mathrm{N}$; $\mathrm{j} \in 2 \mathrm{NU}\{0\}=\{0,2,4, \ldots\} \mathrm{z} \in \mathrm{U}^{*}$. or, equivalently, if:

$$
\left|\begin{array}{c}
\frac{z\left(\left(f^{*} g\right)(z)\right)^{(j+1)}}{\left(\left(f^{*} g\right)(z)\right)^{(j)}}+(p+j) \\
B \frac{z\left(\left(f^{*} g\right)(z)\right)^{(j+1)}}{\left(\left(f^{*} g\right)(z)\right)^{(j)}} \\
+[B(p+j)-(p+j-\alpha)(A-B)]
\end{array}\right|<1
$$

The aim intended to be achieved in the current analysis is to identify coefficient estimates, distortion theorem, radii of meromorphically $p$-valent star likeness and convexity of order $(0 \leq \gamma<p)$, neighborhood property, partial sums. Moreover, the convolution properties and integral operator and integral representation are investigated.

\section{Upper Bounds}

In the following section, we establisha characterization property which provides a necessary, sufficient condition for a function $f(z)$, defined by (1.1), belongs to the class $\mathcal{S}_{g}^{*}(A, B, \alpha, p, j)$ and obtain the coefficient estimates.
Theorem 1. Let the function $f(z)$ be given by (1.1). Then $f(z) \in \mathcal{S}_{g}^{*}(A, B, \alpha, p, j)$ if and only if:

$$
\begin{aligned}
& \sum_{n=p}^{\infty} \delta(n, j)[(1+B)(n+p)+(p+j-\alpha)(B-A)] \\
& a_{n} b_{n} \leq(p+j-\alpha)(B-A) \frac{(p+j-1) !}{(p-1) !}
\end{aligned}
$$

Where:

$$
\delta(n, j)=\frac{n !}{(n-j) !}=\left\{\begin{array}{lr}
n(n-1) \ldots(n-j+1) & (j \neq 0) \\
1 & (j=0)
\end{array}\right.
$$

Proof. Suppose that the function $f(z)$ defined by (1.1) is in the class $\mathcal{S}_{g}^{*}(A, B, \alpha, p, j)$. Then from (1.1) and (1.4), we have:

$$
\begin{aligned}
& \left|\begin{array}{l}
\frac{z\left(f^{*} g\right)^{(j+1)}(z)+(p+j)\left(f^{*} g\right)^{(j)}(z)}{B z\left(f^{*} g\right)^{(j+1)}(z)+[B(p+j)} \\
-(p+j-\alpha)(A-B)]\left(f^{*} g\right)^{(j)}(z)
\end{array}\right| \\
& =\left|\begin{array}{c}
-\sum_{n=p}^{\infty} \delta(n, j)(n+p) a_{n} b_{n} z^{n+p} \\
(p+j-\alpha)(B-A) \frac{(p+j-1) !}{(p-1) !}-\sum_{n=p}^{\infty} \delta(n, j) \\
{[B(n+p)-(p+j-\alpha)(B-A)] a_{n} b_{n} z^{n+p}}
\end{array}\right| \\
& <1\left(z \in U^{*}\right)
\end{aligned}
$$

The choice of $z$ to be real and letting $\mathrm{z} \rightarrow 1^{-}$through real value when $|\operatorname{Re}\{z\}| \leq|z|$ for any $z$, then the inequality (2.2) directly gives the desired condition in (2.1). Conversely, assume that the condition (2.1) holds true and let $|z|=1$, then we have:

$$
\begin{aligned}
& \left|\begin{array}{l}
\frac{z\left(f^{*} g\right)^{(j+1)}(z)+(p+j)\left(f^{*} g\right)^{(j)}(z)}{B z\left(f^{*} g\right)^{(j+1)}(z)+[B(p+j)} \\
-(p+j-\alpha)(A-B)]\left(f^{*} g\right)^{(j)}(z)
\end{array}\right| \\
& \leq \frac{\sum_{n=p}^{\infty} \delta(n, j)(n+p) a_{n} b_{n}}{(p+j-\alpha)(B-A) \frac{(p+j-1) !}{(p-1) !}-\sum_{n=p}^{\infty} \delta(n, j)} \\
& \quad[B(n+p)-(p+j-\alpha)(B-A)] a_{n} b_{n} \\
& <1\left(z \in U^{*}\right)
\end{aligned}
$$


By hypothesis. Hence, by the maximum modulus theorem $f(z) \in \mathcal{S}_{g}^{*}(A, B, \alpha, p, j)$.

Corollary 1. Let $f(z)$ be defined by (1.1). If $f(z) \in \mathcal{S}_{g}^{*}(A, B, \alpha, p, j)$, then:

$$
\begin{gathered}
a_{n} \leq \frac{(p+j-\alpha)(B-A) \frac{(p+j-1) !}{(p-1) !}}{\delta(n, j)[(1+B)(n+p)+(p+j-\alpha)(B-A)] b_{n}} \\
(j \leq p \leq n, j \in 2 \mathbb{N U}\{0\}, p \in \mathbb{N})
\end{gathered}
$$

The result is sharp for the function $f(z)$ given by:

$$
\begin{gathered}
f(z)=z^{-p}-\frac{(p+j-\alpha)(B-A) \frac{(p+j-1) !}{(p-1) !}}{\delta(n, j)\left[\begin{array}{l}
(1+B)(n+p) \\
+(p+j-\alpha)(B-A)
\end{array}\right] b_{n}} z^{n} \\
(j \leq p \leq n, j \in 2 \mathbb{N} \mathbb{U}\{0\}, p \in \mathbb{N})
\end{gathered}
$$

\section{Distortion Theorem}

The following theorem proves the distortion inequality for the function $f \in \mathcal{S}_{g}^{*}(A, B, \alpha, p, j)$. Such property for another class is investigated by many researchers among them Aouf and El-Ashwah (2009).

Theorem 2: If a function $f(z)$ given by (1.1) is in the class $\mathcal{S}_{g}^{*}(A, B, \alpha, p, j)$. Then:

$$
\begin{aligned}
& \frac{(p+q-1) !}{(p-1) !} r^{-p-q} \\
& -\frac{(p+j-\alpha)(B-A) \frac{(p+j-1) !}{(p-1) !}}{\frac{(p-q) !}{(p-j) !}[(1+B) 2 p+(p+j-\alpha)(B-A)] b_{p}} \\
& \leq \frac{r^{n-q} \leq\left|f^{(q)}(z)\right|}{(p+q-1) !} r^{-p-q} \\
& +\frac{(p+j-\alpha)(B-A) \frac{(p+j-1) !}{(p-1) !}}{\frac{(p-q) !}{(p-j) !}[(1+B) 2 p+(p+j-\alpha)(B-A)] b_{p}} r^{n-q} \\
& \left(0<|z|=r<1 ; 0 \leq q \leq j \leq p ; q \in \mathbb{N}_{0} ; j \in 2 \mathbb{N} \mathbb{U}\{0\} ; p \in \mathbb{N}\right)
\end{aligned}
$$

Provided $b_{n} \geq b_{p} \geq 1(n \geq p)$. The result is sharp for the functions $f$ given by:

$$
\begin{aligned}
& f(z)=z^{-p} \\
& -\frac{(p+j-\alpha)(B-A) \frac{(p+j-1) !}{(p-1) !}}{\delta(p, j)[(1+B) 2 p+(p+j-\alpha)(B-A)] b_{p}} z^{p}
\end{aligned}
$$

$$
(j \leq p \leq n, j \in 2 \mathbb{N} U\{0\}, p \in \mathbb{N})
$$

Proof. Since $f(z)=z^{-p}-\sum_{n=p}^{\infty} a_{n} z^{n}$ is in the class $\mathcal{S}_{g}^{*}(A, B, \alpha, p, j)$ and from Theorem 1 together with:

$$
\begin{aligned}
& \frac{(p-q) !}{(p-j) !}[(1+B) 2 p+(p+j-\alpha)(B-A)] b_{p} \\
& \leq \frac{(n-q) !}{(n-j) !}[(1+B) 2 p+(p+j-\alpha)(B-A)] b_{n}(n \geq p)
\end{aligned}
$$

We have:

$$
\begin{aligned}
& \frac{(p-q) !}{(p-j) !}[(1+B) 2 p+(p+j-\alpha)(B-A)] b_{p} \\
& \sum_{n=p}^{\infty} \frac{n !}{(n-q) !} a_{n} \leq(p+j-\alpha)(B-A) \frac{(p+j-1) !}{(p-1) !}
\end{aligned}
$$

Or:

$\sum_{n=p}^{\infty} \frac{n !}{(n-q) !} a_{n} \leq \frac{(p+j-\alpha)(B-A) \frac{(p+j-1) !}{(p-1) !}}{\frac{(p-q) !}{(p-j) !}[(1+B) 2 p+(p+j-\alpha)(B-A)] b_{p}}$

Now by differentiating $f(z)=z^{-p}-\sum_{n=p}^{\infty} a_{n} z^{n}(q)$ times, we have:

$$
\begin{aligned}
& f^{(q)}(z)=(-1)^{q} \frac{(p+q-1) !}{(p-1) !} z^{-p-q}-\sum_{n=p}^{\infty} \frac{n !}{(n-q) !} \\
& a_{n} z^{n-q}(j \leq p \leq n, j \in 2 \mathbb{N U}\{0\}, p \in \mathbb{N})
\end{aligned}
$$

We have for $|z|=r<1$.

$$
\begin{aligned}
\left|f^{(q)}(z)\right| \leq \frac{(p+q-1) !}{(p-1) !} r^{-p-q}+r^{n-q} \sum_{n=p}^{\infty} \frac{n !}{(n-q) !} a_{n} r^{n-q} \\
\quad \leq \frac{(p+q-1) !}{(p-1) !} r^{-p-q}+r^{n-q} \\
\\
\quad \frac{(p+j-\alpha)(B-A) \frac{(p+j-1) !}{(p-1) !}}{(p-j) !}[(1+B) 2 p+(p+j-\alpha)(B-A)] b_{p}
\end{aligned}
$$


And similarly:

$$
\begin{aligned}
& \left|f^{(q)}(z)\right| \geq \frac{(p+q-1) !}{(p-1) !} r^{-p-q}-r^{n-q} \\
& \frac{(p+j-\alpha)(B-A) \frac{(p+j-1) !}{(p-1) !}}{\frac{(p-q) !}{(p-j) !}[(1+B) 2 p+(p+j-\alpha)(B-A)] b_{p}}
\end{aligned}
$$

The sharpness of the inequality in (3.1) satisfies by the function $f(z)$ given by (3.2).

\section{Radii of Starlikeness and Convexity}

This section considers the radii of meromorphically $\mathrm{p}$-valent starlikeness of order $\gamma(0 \leq \gamma<p)$ and meromorphically p-valent convexity of order $\gamma(0 \leq \gamma<p)$ for the functions that belong to the class $\mathcal{S}_{g}^{*}(A, B, \alpha, p, j)$, by using methods applied by Kamali et al. (2011) and others.

Theorem 3: Let $f(z) \in \mathcal{S}_{g}^{*}(A, B, \alpha, p, j)$. Then:

$f$ is meromorphically $p$-valent starlike of order $\gamma(0 \leq \gamma<p)$ in $|z|<r_{2}$, where:

$$
r_{1}=\inf _{n \geq p}\left\{\frac{(p-\gamma) \delta(n, j)\left[\begin{array}{l}
(1+B)(p+n) \\
+(p+j-\alpha)(B-A)
\end{array}\right] b_{n}}{(n+\gamma)(p+j-\alpha)(B-A) \frac{(p+j-1) !}{(p-1) !}}\right\}
$$

$f$ is meromorphically $p$-valent convex of order $\gamma(0 \leq \gamma<p)$ in $|z|<r_{2}$, where:

$$
r_{2}=\inf _{n \geq p}\left\{\frac{p(p-\gamma) \delta(n, j)\left[\begin{array}{l}
(1+B)(p+n) \\
+(p+j-\alpha)(B-A)
\end{array}\right]}{b_{n}}\right\}^{\frac{1}{n+p}}
$$

Each of these results is sharp for the function $f(z)$ given by (2.5).

Proof. (i) From the definition (1.1), we obtain:

$$
\left|\frac{\frac{z f^{\prime}(z)}{f(z)}+p}{\frac{z f^{\prime}(z)}{f(z)}-p+2 \gamma}\right| \leq \frac{\sum_{n=p}^{\infty}(n+p) a_{n}|z|^{n+p}}{2(p-\gamma)-\sum_{n=p}^{\infty}(n-p+2 \gamma) a_{n}|z|^{n+p}}
$$

Thus, we have the desired inequality:

$$
\left|\frac{\frac{z f^{\prime}(z)}{f(z)}+p}{\frac{z f^{\prime}(z)}{f(z)}-p+2 \gamma}\right| \leq 1(0 \leq \gamma<p ; p \in \mathbb{N})
$$

If:

$\sum_{n=p}^{\infty} \frac{(n+\gamma)}{(p-\gamma)} a_{n}|z|^{n+p} \leq 1$

So, by Theorem 1, the condition (4.3) will be true if:

$$
\frac{(n+\gamma)}{(p-\gamma)}|z|^{n+p} \leq \frac{\delta(n, j)\left[\begin{array}{l}
(1+B)(p+n) \\
+(p+j-\alpha)(B-A)
\end{array}\right]}{(p+j-\alpha)(B-A) \frac{(p+j-1) !}{(p-1) !}},(p \leq n ; p \in \mathbb{N})
$$

Therefore:

$|z| \leq\left\{\frac{(p-\gamma) \delta(n, j)\left[\begin{array}{l}(1+B)(p+n) \\ +(p+j-\alpha)(B-A)\end{array}\right]}{b_{n}}\right\}^{\frac{1}{n+p}}$

Putting $|z|=r_{1}$ in (4.4), we get the radius of starlikeness.

In order to prove the second assertion of Theorem 3, we find from the definition (1.1) that:

$$
\left|\frac{1+\frac{z f^{\prime \prime}(z)}{f^{\prime}(z)}+p}{1+\frac{z f^{\prime \prime}(z)}{f^{\prime}(z)}-p+2 \gamma}\right| \leq \frac{\sum_{n=p}^{\infty} n(n+p) a_{n}|z|^{n+p}}{2 p(p-\gamma)-\sum_{n=p}^{\infty} n(n-p+2 \gamma) a_{n}|z|^{n+p}}
$$

Thus, we have the desired inequality:

$$
\left|\frac{1+\frac{z f^{\prime \prime}(z)}{f^{\prime}(z)}+p}{1+\frac{z f^{\prime \prime}(z)}{f^{\prime}(z)}-p+2 \gamma}\right| \leq 1,(0 \leq \gamma<p ; p \in \mathbb{N})
$$

If:

$$
\sum_{n=p}^{\infty} \frac{n(n+\gamma)}{p(p-\gamma)} a_{n}|z|^{n+p} \leq 1
$$

Hence, by Theorem 1, the condition (4.5) will be satisfied if: 


$$
\begin{aligned}
& \frac{n(n+\gamma)}{p(p-\gamma)}|z|^{n+p} \leq \frac{\delta(n, j)[(p+j-\alpha)(B-A)+(1+B)(p+n)] b_{n}}{(p+j-\alpha)(B-A) \frac{(p+j-1) !}{(p-1) !}} \\
& (p \leq n, p \in \mathbb{N})
\end{aligned}
$$

Therefore:

$$
|z| \leq\left\{\frac{p(p-\gamma) \delta(n, j)\left[\begin{array}{l}
(p+j-\alpha) \\
(B-A)+(1+B)(p+n)
\end{array}\right]}{n(n+\gamma)(p+j-\alpha)(B-A) \frac{(p+j-1) !}{(p-1) !}}\right\}^{\frac{1}{n+p}}
$$

Setting $|z|=r_{1}$ in (4.6), we obtain the radius of convexity, which completes the proof of Theorem 3.

\section{Neighborhood Property}

Depending on the earlier works by (Goodman, 1957; Ruscheweyh, 1981; Liu and Srivastava, 2004; Aouf, 2009; Aouf and El-Ashwah, 2009) that based upon the familiar concept of neighborhood of analytic functions, we introduce the definition of the $\delta$-neighborhood of a function $f(z) \in \mathcal{S}_{g}^{*}(A, B, \alpha, p, j)$ by:

$$
\begin{aligned}
N_{\delta}(f)=\left\{\begin{array}{l}
\phi \in \mathcal{S}_{g}^{*}(A, B, \alpha, p, j): \phi(z)=z^{-p}-\sum_{n=p}^{\infty} d_{n} z^{n} \\
\text { and } \sum_{n=p}^{\infty} \frac{\delta(n, j)[(1+B)(n+p)+(p+j-\alpha)(B-A)] b_{n}}{(p+j-\alpha)(B-A) \frac{(p+j-1) !}{(p-1) !}} \\
\left|a_{n}-d_{n}\right| \leq \delta
\end{array}\right\} \\
\left(\begin{array}{l}
-1 \leq A<B \leq 1 ; 0<B \leq 1 ; 0 \leq \alpha<p \\
-j ; j \leq p \leq n ; p \in N, ; j \in 2 \mathbb{N U}\{0\} ; \delta>0
\end{array}\right)
\end{aligned}
$$

Theorem 4: If $f(z) \in \mathcal{S}_{g}^{*}(A, B, \alpha, p, j)$ satisfies the following condition $\frac{f(z)+\sigma z^{-p}}{1+\sigma} \in \mathcal{S}_{g}^{*}(A, B, \alpha, p, j)$ for any complex number $\sigma,|\sigma|=1$ such that $|\sigma|<\delta$, then $N_{\delta}(f) \subset \mathcal{S}_{g}^{*}(A, B, \alpha, p, j)$.

Proof. It is easily obvious that $f(z) \in \mathcal{S}_{g}^{*}(A, B, \alpha, p, j)$ if and only if:

$$
\left|\frac{z\left(f^{*} g\right)^{(j+1)}(z)+(p+j)\left(f^{*} g\right)^{(j)}(z)}{B z\left(f^{*} g\right)^{(j+1)}(z)+[B(p+j)-(p+j-\alpha)(A-B)]\left(f^{*} g\right)^{(j)}(z)}\right|<1
$$

For any complex number $\varepsilon$ with $|\varepsilon|=$, we have:

$\frac{z\left(f^{*} g\right)^{(j+1)}(z)+(p+j)\left(f^{*} g\right)^{(j)}(z)}{B z\left(f^{*} g\right)^{(j+1)}(z)+[B(p+j)+(p+j-\alpha)(B-A)]\left(f^{*} g\right)^{(j)}(z)} \neq \varepsilon$
Consequently:

$$
1-\sum_{n=p}^{\infty} \frac{\delta(n, j)\left[\begin{array}{l}
(\varepsilon B-1)(n+p) \\
+\varepsilon(p+j-\alpha)(B-A)
\end{array}\right] a_{n} b_{n}}{\varepsilon(p+j-\alpha)(B-A) \frac{(p+j-1) !}{(p-1) !}} z^{n+p} \neq 0
$$

Which is equivalent to:

$$
z^{-p}-\sum_{n=p}^{\infty} \frac{\delta(n, j)[(\varepsilon B-1)(n+p)+\varepsilon(p+j-\alpha)(B-A)] a_{n} b_{n}}{\varepsilon(p+j-\alpha)(B-A) \frac{(p+j-1) !}{(p-1) !}} z^{n} \neq 0
$$

Thus, $\quad f(z) \in \mathcal{S}_{g}^{*}(A, B, \alpha, p, j) \quad$ if and only if $\frac{\left(f^{*} h\right)(z)}{z^{-p}} \neq 0\left(z \in U^{*}\right)$, where $h(z)=z^{-p}-\sum_{n=p}^{\infty} c_{n} z^{n} \quad$ and $c_{n}=\frac{\delta(n, j)[(\varepsilon B-1)(n+p)+\varepsilon(p+j-\alpha)(B-A)] b_{n}}{\varepsilon(p+j-\alpha)(B-A) \frac{(p+j-1) !}{(p-1) !}}, \quad$ we have $\left|c_{n}\right| \leq \frac{\delta(n, j)[(1+B)(n+p)+(p+j-\alpha)(B-A)] b_{n}}{(p+j-\alpha)(B-A) \frac{(p+j-1) !}{(p-1) !}}$. Since $\frac{f(z)+\sigma z^{-p}}{1+\sigma} \in \mathcal{S}_{g}^{*}(A, B, \alpha, p, j)$, therefore $\frac{\left(\frac{f(z)+\sigma z^{-p}}{1+\sigma} * h(z)\right)}{z^{-p}} \neq 0$, which is equivalent to: $\frac{(f * h)(z)}{(1+\sigma) z^{-p}}+\frac{\sigma}{1+\sigma} \neq 0$

Now suppose that $\left|\frac{\left(f^{*} h\right)(z)}{z^{-p}}\right|<\delta$ Then by (5.1), we have:

$\left|\frac{\left(f^{*} h\right)(z)}{(1+\sigma) z^{-p}}+\frac{\sigma}{1+\sigma}\right| \geq \frac{|\sigma|}{|1+\sigma|}-\frac{1}{|1+\sigma|}\left|\frac{\left(f^{*} h\right)(z)}{z^{-p}}\right|>\frac{|\sigma|-\delta}{|1+\sigma|} \geq 0$

This is contradiction by $|\sigma|<\delta$ and however, we have $\left|\frac{\left(f^{*} h\right)(z)}{z^{-p}}\right| \geq \delta$. If $\phi(z)=z^{-p}-\sum_{n=p}^{\infty} d_{z^{2}} z^{n} \in N_{\delta}(f)$ then:

$$
\delta-\left|\frac{\left(\phi^{*} h\right)(z)}{z^{-p}}\right| \leq\left|\frac{\left((\phi-f)^{*} h\right)(z)}{z^{-p}}\right| \leq \sum_{n=p}^{\infty}\left|a_{n}-d_{n}\right|\left|c_{n}\right|\left|z^{n}\right|
$$

$<\sum_{n=p}^{\infty} \frac{\delta(n, j)[(1+B)(n+p)+(p+j-\alpha)(B-A)] b_{n}}{(p+j-\alpha)(B-A) \frac{(p+j-1) !}{(p-1) !}}\left|a_{n}-d_{n}\right| \leq \delta$ 


\section{Partial Sums}

By following (Silvia, 1985; Silverman, 1997) previous investigations, we are able in this section to identify the bounds $\frac{f(z)}{s_{p+k}(z)}$ and $\frac{s_{p+k}(z)}{f(z)}$.

Theorem 5: Let $f(z) \in \Sigma^{*}(p)$ be in the class $\mathcal{S}_{g}^{*}(A, B, \alpha, p, j)$ and define the partial sums as follows:

$$
\begin{aligned}
& S_{1}(z)=z^{-p} \\
& S_{m}(z)=z^{-p}-\sum_{n=p}^{m} a_{n} z^{n}(m \geq p)
\end{aligned}
$$

Also suppose that:

$\sum_{n=p}^{\infty} d_{n} a_{n} \leq 1\left(d_{n}=\frac{\delta(n, j)\left[\begin{array}{l}(1+B)(n+p) \\ +(p+j-\alpha)(B-A)\end{array}\right]}{b_{n}}\right)$

Then we have:

$\operatorname{Re}\left\{\frac{f(z)}{s_{m}(z)}\right\}>1-\frac{1}{d_{m+1}}$

$\operatorname{Re}\left\{\frac{s_{m}(z)}{f(z)}\right\}>\frac{d_{m+1}}{1+d_{m+1}}\left(z \in U^{*}\right)$

The results in (6.2) and (6.3) are sharp for $n$.

Proof. We can see from (6.1) that $1<d_{n}<d_{n}+1 \quad(n \in N)$. Since $\left\{d_{n}\right\}$ is an increasing sequence, we obtain:

$\sum_{n=p}^{m} a_{n}+d_{m+1} \sum_{n=m+1}^{\infty} a_{n} \leq 1$

By setting

$g_{1}(z)=d_{m+1}\left(\frac{f(z)}{s_{m}(z)}-\left(1-\frac{1}{d_{m+1}}\right)\right)=1-\frac{d_{m+1} \sum_{n=p}^{\infty} a_{n} z^{n+p}}{1-\sum_{n=p}^{m} a_{n} z^{n+p}}$ from (6.4) we find that $\left|\frac{g_{1}(z)-1}{g_{1}(z)+1}\right| \leq \frac{d_{m+1} \sum_{n=p}^{\infty} a_{n} z^{n+p}}{2-2 \sum_{n=p}^{m} a_{n}-d_{p+k} \sum_{n=p+k}^{\infty} a_{n}} \leq 1$.

This proves (6.2). If we take $f(z)=z^{-p}-\frac{z^{p+k}}{d_{m+1}}$ then $\frac{f(z)}{s_{m}(z)}=1-\frac{z^{2 p+k}}{d_{m+1}} \rightarrow 1-\frac{1}{d_{m+1}}$ as $z \rightarrow 1$ which shows that the bound in (6.2) is best possible.
Similary,

if

we

let

$g_{2}(z)=\left(1+d_{m+1}\right)\left(\frac{s_{m}(z)}{f(z)}-\frac{d_{m+1}}{1+d_{m+1}}\right)=1+\frac{\left(1+d_{m+1}\right) \sum_{n=m+1}^{\infty} a_{n} z^{n+p}}{1-\sum_{n=p}^{\infty} a_{n} z^{n+p}}$

and make use of (6.4), we obtain $\left|\frac{g_{2}(z)-1}{g_{2}(z)+1}\right| \leq \frac{\left(1+d_{m+1}\right) \sum_{n=m+1}^{\infty} a_{n}}{2-2 \sum_{n=p}^{m} a_{n}-\left(d_{m+1}-1\right) \sum_{n=m+1}^{\infty} a_{n}} \leq 1,\left(z \in U^{*}\right)$

which yields inequality (6.3). If we take $f(z)=z^{-p}-\frac{z^{p+k}}{d_{m+1}}$ then $\frac{s_{m}(z)}{f(z)}=\frac{d_{m+1}}{d_{m+1}-z^{2 p+k}} \rightarrow \frac{d_{m+1}}{d_{m+1}-1}$ as $z \rightarrow 1$ which shows that the bound in (6.3) is best possible.

\section{Convolution Properties}

This section concentrates on a way to derive the convolution properties by using Schild and Silverman (1975) techniques. At the beginning, let's recall the following definition: Let the functions $f_{i}(z)(i=1,2)$ be defined by:

$$
f_{i}(z)=z^{-p}-\sum_{n=p}^{\infty} a_{n, i} z^{n}\left(a_{n} \geq 0 ; p \in \mathbb{N} ; i=1,2\right)
$$

The modified Hadamard product (or convolution) of $f_{1}(z)$ and $f_{2}(z)$ is defined by:

$$
\left(f_{1} * f_{2}\right)(z)=z^{-p}-\sum_{n=p}^{\infty} a_{n, i} z^{n}
$$

Theorem 6: Let the functions $f_{1}(z)(i=1,2)$ defined by (7.1) be in the class $\mathcal{S}_{g}^{*}(A, B, \alpha, p, j)$ and $b_{n} \geq b_{p} \geq 1(n \geq p)$. Then

$\left(f_{1}^{*} f_{2}\right)(z) \in \mathcal{S}_{g}^{*}(A, B, \gamma, p, j)$,

$$
\begin{gathered}
\text { where } \gamma=p+j-\frac{(p+j-\alpha)^{2}(B-A)(2 p) \frac{(p+j-1) !}{(p-1) !}}{\delta(p, j)[(1+B)(2 p)+(p+j-\alpha)(B-A)]^{2}} . \\
b_{p}-(p+j-\alpha)^{2}(B-A)^{2} \frac{(p+j-1) !}{(p-1) !}
\end{gathered}
$$

The result is sharp for the functions $f_{\mathrm{i}}(z)(i=1,2)$ given by:

$$
\begin{gathered}
f_{i}(z)=z^{-p}-\frac{(p+j-\alpha)(B-A) \frac{(p+j-1) !}{(p-1) !}}{\delta(p, j)\left[\begin{array}{l}
(1+B) 2 p \\
+(p+j-\alpha)(B-A)
\end{array}\right] b_{p}} z^{p} \\
\left(i=1,2 ; p \in N ; j \in 2 \mathbb{N} U\{0\} ; z \in U^{*}\right)
\end{gathered}
$$

Proof. In view of Theorem 1, it suffices to prove that: 


$$
\begin{aligned}
& \sum_{n=p}^{\infty} \frac{\delta(n, j)[(1+B)(n+p)+(p+j-\gamma)(B-A)]}{(p+j-\gamma)(B-A) \frac{(p+j-1) !}{(p-1) !}} \\
& a_{n, 1} a_{n, 2} b_{n} \leq 1,(p \in N ; j \in 2 \mathbb{N} U\{0\})
\end{aligned}
$$

Since $f_{i}(z) \in \mathcal{S}_{g}^{*}(A, B, \alpha, p, j)(i=1,2)$, we have:

$$
\begin{aligned}
& \sum_{n=p}^{\infty} \frac{\delta(n, j)[(1+B)(n+p)+(p+j-\alpha)(B-A)]}{(p+j-\alpha)(B-A) \frac{(p+j-1) !}{(p-1) !}} \\
& a_{n, i} b_{n} \leq 1,(i=1,2 ; p \in N ; j \in 2 \mathbb{N} U\{0\})
\end{aligned}
$$

Therefore, by the Cauchy-Schwarz inequality, we obtain:

$$
\begin{aligned}
& \sum_{n=p}^{\infty} \frac{\delta(n, j)[(1+B)(n+p)+(p+j-\alpha)(B-A)]}{(p+j-\alpha)(B-A) \frac{(p+j-1) !}{(p-1) !}} \\
& \sqrt{a_{n, 1} a_{n, 2}} b_{n} \leq 1(p \in N ; j \in 2 \mathbb{N} U\{0\})
\end{aligned}
$$

Hence, we need to show that

$$
\begin{aligned}
& \frac{\delta(n, j)[(1+B)(n+p)+(p+j-\gamma)(B-A)]}{(p+j-\gamma)(B-A) \frac{(p+j-1) !}{(p-1) !}} a_{n, 1} a_{n, 2} b_{n} \\
& \leq \frac{\delta(n, j)[(1+B)(n+p)+(p+j-\alpha)(B-A)]}{(p+j-\alpha)(B-A) \frac{(p+j-1) !}{(p-1) !}} \sqrt{a_{n, 1} a_{n, 2}} b_{n}, \\
& (n \geq p ; p \in N ; j \in 2 \mathbb{N U}\{0\})
\end{aligned}
$$

Or, equivalently, that:

$$
\sqrt{a_{n, 1} a_{n, 2}} \leq \frac{[(1+B)(n+p)+(p+j-\alpha)(B-A)](p+j-\gamma)}{[(1+B)(n+p)+(p+j-\gamma)(B-A)](p+j-\alpha)}
$$$$
(n \geq p ; p \in N ; j \in 2 \mathbb{N} \mathbb{U}\{0\})
$$

From the equality (7.4), we have

$$
\begin{aligned}
& \sqrt{a_{n, 1} a_{n, 2}} \leq \frac{(p+j-\alpha)(B-A) \frac{(p+j-1) !}{(p-1) !}}{\delta(n, j)[(1+B)(n+p)+(p+j-\alpha)(B-A)] b_{n}}, \\
& (n \geq p ; p \in N ; j \in 2 \mathbb{N U}\{0\})
\end{aligned}
$$

So, it is sufficient to prove that:

$$
\begin{gathered}
\frac{(p+j-\alpha)(B-A) \frac{(p+j-1) !}{(p-1) !}}{\delta(n, j)[(1+B)(n+p)+(p+j-\alpha)(B-A)] b_{n}} \\
\leq \frac{[(1+B)(n+p)+(p+j-\alpha)(B-A)](p+j-\gamma)}{[(1+B)(n+p)+(p+j-\gamma)(B-A)](p+j-\alpha)},
\end{gathered}
$$

$(n \geq p ; p \in N ; j \in 2 \mathbb{N U}\{0\})$

It is follows from (7.5) that:

$$
\begin{aligned}
& \gamma \leq p+j- \frac{(p+j-\alpha)^{2}(B-A)(n+p) \frac{(p+j-1) !}{(p-1) !}}{\delta(n, j)[(1+B)(n+p)+(p+j-\alpha)(B-A)]^{2}} \\
& b_{n}-(p+j-\alpha)^{2}(B-A)^{2} \frac{(p+j-1) !}{(p-1) !}
\end{aligned}
$$

Now, defining the function $\varphi(n)$ by:

$$
\begin{gathered}
\varphi(n)=p+j-\frac{(p+j-\alpha)^{2}(B-A)(n+p) \frac{(p+j-1) !}{(p-1) !}}{\delta(n, j)[(1+B)(n+p)+(p+j-\alpha)(B-A)]^{2}} \\
b_{n}-(p+j-\alpha)^{2}(B-A)^{2} \frac{(p+j-1) !}{(p-1) !}
\end{gathered}
$$

We easily see that $\varphi(n)$ is an increasing function of $n$. So we have:

$$
\begin{aligned}
& \gamma \leq \varphi(p)=p+j- \frac{(p+j-\alpha)^{2}(B-A)(2 p) \frac{(p+j-1) !}{(p-1) !}}{\delta(p, j)[(1+B)(2 p)+(p+j-\alpha)(B-A)]^{2}} \\
& b_{p}-(p+j-\alpha)^{2}(B-A)^{2} \frac{(p+j-1) !}{(p-1) !}
\end{aligned}
$$

Which completes the proof of Theorem 6 .

Theorem 7: Let the function $f_{1}(z)$ defined by $(7.1)$ be in the class $\mathcal{S}_{g}^{*}(A, B, \varepsilon, p, j)$ and the function $f_{2}(z)$ defined by (7.1) be in the class $\mathcal{S}_{g}^{*}(A, B, \varepsilon, p, j)$ provided $b_{n} \geq b_{p} \geq 1(n \geq p)$. Then $\left(f_{1}^{*} f_{2}\right)(z) \in \mathcal{S}_{g}^{*}(A, B, \theta, p, j)$, where:

$$
\begin{gathered}
\theta=p+j-\frac{(p+j-\alpha)(p+j-\varepsilon)(B-A)(2 p) \frac{(p+j-1) !}{(p-1) !}}{\delta(n, j) \mu(\alpha) \mu(\varepsilon) b_{p}-(p+j-\alpha)} \\
(p+j-\varepsilon)(B-A)^{2} \frac{(p+j-1) !}{(p-1) !}
\end{gathered}
$$

Where:

$$
\mu(\rho)=[(1+B)(2 p)+(p+j-\rho)(B-A)]
$$


The result is sharp for the functions $f_{i}(z)(i=1,2)$ given $\mathrm{b}$ :

$$
f_{1}(z)=z^{-p}-\frac{(p+j-\alpha)(B-A) \frac{(p+j-1) !}{(p-1) !}}{\delta(p, j)[(1+B) 2 p+(p+j-\alpha)(B-A)] b_{p}} z^{p}
$$$$
\left(n \geq p ; p \in N ; j \in 2 \mathbb{N U}\{0\} ; z \in U^{*}\right)
$$$$
f_{2}(z)=z^{-p}-\frac{(p+j-\varepsilon)(B-A) \frac{(p+j-1) !}{(p-1) !}}{\delta(p, j)[(1+B) 2 p+(p+j-\varepsilon)(B-A)] b_{p}} z^{p}
$$

$\left(n \geq p ; p \in N ; j \in 2 \mathbb{N U}\{0\} ; z \in U^{*}\right)$

Theorem 8: Let the functions $f_{i}(z)(i=1,2)$ defined by (7.1) be in the class $\mathcal{S}_{g}^{*}(A, B, \varepsilon, p, j)$ and $b_{n} \geq b_{p} \geq 1(n \geq p)$. Then the function $h(z)$ defined by:

$$
h(z)=z^{-p}-\sum_{n=p}^{\infty}\left(a_{n, 1}^{2}+a_{n, 2}^{2}\right) z^{n}
$$

Belongs to the class $\mathcal{S}_{g}^{*}(A, B, \varepsilon, p, j)$, where:

$$
\begin{gathered}
\omega=p+j-\frac{2(p+j-\alpha)^{2}(B-A)(2 p) \frac{(p+j-1) !}{(p-1) !}}{\delta(n, j)[(1+B)(2 p)+(p+j-\alpha)(B-A)]^{2}} \\
b_{n}-2(p+j-\alpha)^{2}(B-A)^{2} \frac{(p+j-1) !}{(p-1) !}
\end{gathered}
$$

The result is sharp for the functions $f_{i}(z)(i=1,2)$ given by (7.3).

Proof. Since $f_{i}(z) \in \mathcal{S}_{g}^{*}(A, B, \alpha, p, j)(i=1,2)$. Thus, by Theorem 1:

$$
\begin{aligned}
& {\left[\sum_{n=p}^{\infty} \frac{\delta(n, j)[(1+B)(n+p)+(p+j-\alpha)(B-A)]}{(p+j-\alpha)(B-A) \frac{(p+j-1) !}{(p-1) !}} a_{n, i} b_{n}\right]^{2}} \\
& \leq 1,(i=1,2 ; p \in N ; j \in 2 \mathbb{N U}\{0\})
\end{aligned}
$$

Note that:

$$
\begin{aligned}
& \sum_{n=p}^{\infty}\left[\frac{\delta(n, j)[(1+B)(n+p)+(p+j-\alpha)(B-A)]}{\left.(p+j-\alpha)(B-A) \frac{(p+j-1) !}{(p-1) !}\right]_{n, i}^{2}} a_{n}^{2} b_{n}^{2}\right. \\
& \leq\left[\sum_{n=p}^{\infty} \frac{\delta(n, j)[(1+B)(n+p)+(p+j-\alpha)(B-A)]}{(p+j-\alpha)(B-A) \frac{(p+j-1) !}{(p-1) !}} a_{n, i} b_{n}\right]^{2} \leq 1(7.6) \\
& (i=1,2 ; p \in N ; j \in 2 \mathbb{N U}\{0\})
\end{aligned}
$$

Implies that:

$$
\begin{aligned}
& \sum_{n=p}^{\infty} \frac{1}{2}\left[\frac{\delta(n, j)[(1+B)(n+p)+(p+j-\alpha)(B-A)]}{(p+j-\alpha)(B-A) \frac{(p+j-1) !}{(p-1) !}}\right]^{2} \\
& \left(a_{n, 1}^{2}+a_{n, 2}^{2}\right) b_{n}^{2} \leq 1, \quad(i=1,2 ; p \in N ; j \in 2 \mathbb{N U}\{0\})
\end{aligned}
$$

So, we need to find the largest $\omega$ such that:

$$
\begin{aligned}
& \frac{\delta(n, j)[(1+B)(n+p)+(p+j-\omega)(B-A)]}{(p+j-\omega)(B-A) \frac{(p+j-1) !}{(p-1) !}} b_{n} \\
& \leq \frac{1}{2}\left[\frac{\delta(n, j)[(1+B)(n+p)+(p+j-\alpha)(B-A)]}{(p+j-\alpha)(B-A) \frac{(p+j-1) !}{(p-1) !}} b_{n}\right]^{2}, \\
& (n \geq p ; p \in N ; j \in 2 \mathbb{N U}\{0\})
\end{aligned}
$$

That is:

$$
\begin{gathered}
\omega \leq \leq p+j \frac{2(p+j-\alpha)^{2}(B-A)(n+p) \frac{(p+j-1) !}{(p-1) !}}{\delta(n, j)[(1+B)(n+p)+(p+j-\alpha)(B-A)]^{2}} \\
b_{n}-2(p+j-\alpha)^{2}(B-A)^{2} \frac{(p+j-1) !}{(p-1) !}
\end{gathered}
$$

$(n \geq p ; p \in N ; j \in 2 \mathbb{N U}\{0\})$

Now, defining the function $v(n)$ by:

$$
\begin{aligned}
& v(n)=p+j-\frac{2(p+j-\alpha)^{2}(B-A)(n+p) \frac{(p+j-1) !}{(p-1) !}}{\delta(n, j)[(1+B)(n+p)+(p+j-\alpha)(B-A)]^{2}} \\
& b_{n}-2(p+j-\alpha)^{2}(B-A)^{2} \frac{(p+j-1) !}{(p-1) !} \\
& (n \geq p ; p \in N ; j \in 2 \mathbb{N U}\{0\})
\end{aligned}
$$

It is clear that $\varphi(n)$ is an increasing function of $n$. Thus, we have:

$$
\begin{gathered}
\gamma \leq v(p)=p+j-\frac{2(p+j-\alpha)^{2}(B-A)(2 p) \frac{(p+j-1) !}{(p-1) !}}{\delta(p, j)[(1+B)(2 p)+(p+j-\alpha)(B-A)]^{2}} \\
b_{p}-2(p+j-\alpha)^{2}(B-A)^{2} \frac{(p+j-1) !}{(p-1) !}
\end{gathered}
$$

Which complete the proof of Theorem 9. 


\section{Integral Operator}

By using the techniques presented in (Srivastava et al., 1998; Aouf, 2010) the below theorem shows the obtained integral transforms of functions in the class $\mathcal{S}_{g}^{*}(A, B, \alpha, p, j)$.

Theorem 9: If $f(z) \in \Sigma^{*}(p)$ is in the class $\mathcal{S}_{g}^{*}(A, B, \alpha, p, j)$ and $\quad b_{n} \geq b_{p} \geq 1(n \geq p), \quad$ then the integral transforms:

$$
F_{c+p-1}(z)=c \int_{0}^{1} u^{c+p-1} f(u z) d u,(0<u \leq 1 ; 0<c<\infty)
$$

Are in the class $\mathcal{S}_{g}^{*}(A, B, \alpha, p, j)$, where:

$$
\begin{aligned}
\vartheta=p+j- & \frac{c(p+j-\alpha)(1+B)(2 p)}{(c+2 p)[(1+B)(2 p)+(p+j-\alpha)(B-A)]} \\
& -c(p+j-\alpha)(B-A)
\end{aligned}
$$

The result is the best possible for the function $f(z)$ given by:

$$
\begin{aligned}
& f(z)=z^{-p}-\frac{(p+j-\alpha)(B-A) \frac{(p+j-1) !}{(p-1) !}}{\delta(p, j)[(1+B)(2 p)+(p+j-\alpha)(B-A)] b_{p}} z^{p}, \\
& (p \in N ; j \in 2 \mathbb{N U}\{0\})
\end{aligned}
$$

Proof. Let $f(z)=z^{-p}-\sum_{n=p}^{\infty} a_{n} z^{n} \quad$ in $\quad$ the class $\mathcal{S}_{g}^{*}(A, B, \alpha, p, j)$. Then:

$$
F_{c+p-1}(z)=c \int_{0}^{1} u^{c+p-1} f(u z) d u=z^{-p}-\sum_{n=p}^{\infty} \frac{c}{c+p+n} a_{n} z^{n}
$$

We need to find the largest $\vartheta$ such that:

$$
\sum_{n=p}^{\infty} \frac{c \delta(n, j)[(1+B)(n+p)+(p+j-\vartheta)(B-A)]}{(c+p+n)(p+j-\vartheta)(B-A) \frac{(p+j-1) !}{(p-1) !}} a_{n} b_{n} \leq 1(8.2)
$$

Since $f(z) \in \mathcal{S}_{g}^{*}(A, B, \alpha, p, j)$ and by Theorem 1, we get:

$$
\sum_{n=p}^{\infty} \frac{\delta(n, j)[(1+B)(n+p)+(p+j-\alpha)(B-A)]}{(p+j-\alpha)(B-A) \frac{(p+j-1) !}{(p-1) !}} a_{n} b_{n} \leq 1
$$

Note that the inequality (8.2) is satisfied if:

$$
\begin{aligned}
& \frac{c[(1+B)(n+p)+(p+j-\vartheta)(B-A)]}{(c+p+n)(p+j-\vartheta)} \\
& \leq \frac{[(1+B)(n+p)+(p+j-\alpha)(B-A)]}{(p+j-\alpha)}
\end{aligned}
$$

Or equivalently:

$\vartheta \leq p+j-$

$\frac{c(p+j-\alpha)(1+B)(n+p)}{(c+p+n)[(1+B)(n+p)+(p+j-\alpha)(B-A)]}$

$-c(p+j-\alpha)(B-A)$

Let:

$$
\begin{aligned}
F(n)=p+j- & \frac{c(p+j-\alpha)(1+B)(n+p)}{(c+p+n)[(1+B)(n+p)+(p+j-\alpha)(B-A)]} \\
& -c(p+j-\alpha)(B-A)
\end{aligned}
$$

Then $F_{c+p-1}(n)$ is an increasing function of $n$. Since:

$$
\begin{aligned}
F(p)=p+j- & \frac{c(p+j-\alpha)(1+B)(2 p)}{(c+p+n)[(1+B)(2 p)+(p+j-\alpha)(B-A)]} \\
& -c(p+j-\alpha)(B-A)
\end{aligned}
$$

This completes the proof.

\section{Integral Representation}

In the following theorem, we determine the integral representation of functions that belong to the class $\mathcal{S}_{g}^{*}(A, B, \alpha, p, j)$.

Theorem 10: Let $f(z) \in \Sigma^{*}(p)$ be in the class $\mathcal{S}_{g}^{*}(A, B, \alpha, p, j)$. Then:

$$
\left(f^{*} g\right)^{(j)}(z)=e^{\int^{\tilde{s}} \frac{\varphi(z)[(1+B)(n+p)+(p+j-\alpha)(B-A)]-(p+j)}{z(1-\varphi(z) B}}
$$

where, $|\varphi(t)|<1, z \in U^{*}$ :

Proof. By putting $\frac{z\left(f^{*} g\right)^{(j+1)}(z)}{\left(f^{*} g\right)^{(j)}(z)}=M(z)$ in (1.4), we have:

$$
\left|\frac{M(z)+(p+j)}{B M(z)+[B(p+j)+(p+j-\alpha)(B-A)]}\right|<1
$$

Or equivalently:

$$
\begin{aligned}
& \frac{M(z)+(p+j)}{B M(z)+[B(p+j)+(p+j-\alpha)(B-A)]} \\
& =\varphi(t),|\varphi(t)|<1, z \in U^{*}
\end{aligned}
$$


So:

$$
\frac{\left(f^{*} g\right)^{(j+1)}(z)}{\left(f^{*} g\right)^{(j)}(z)}=\frac{\varphi(z)[B(p+j)+(p+j-\alpha)(B-A)]-(p+j)}{z(1-\varphi(z) B)}
$$

After integration, we get:

$$
\begin{aligned}
& \log \left(\frac{\left(f^{*} g\right)^{(j+1)}(z)}{\left(f^{*} g\right)^{(j)}(z)}\right) \\
& =\int_{0}^{z} \frac{\varphi(z)[B(p+j)+(p+j-\alpha)(B-A)]-(p+j)}{z(1-\varphi(z) B)}
\end{aligned}
$$

Thus:

$$
\frac{\left(f^{*} g\right)^{(j+1)}(z)}{\left(f^{*} g\right)^{(j)}(z)}=e^{\int^{z} \frac{\varphi(z)[B(p+j)+(p+j-\alpha)(B-A)]-(p+j)}{z(1-\varphi(z) B)}}
$$

This completes the proof.

\section{Conclusion}

The purpose of this study was to introduce a certain new subclass of class of meromorphically multivalent functions with negative coefficients. We also investigated the geometric characterization of the coefficient estimates for functions in this subclass. Moreover, we obtained the distortion theorem, radii of meromorphically p-valent starlikeness and convexity, neighborhood property, partial sums, convolution properties, integral operator and integral representation.

\section{Acknowledgment}

The authors are grateful to the referees for their helpful suggestions that improved this article.

\section{Funding Information}

This research is supported by Project No. RG31214AFR from the University of Malaya.

\section{Author's Contributions}

All authors jointly worked on deriving the results and approved the final manuscript.

\section{Ethics}

The authors declare no conflict of interest.

\section{References}

Aouf, M.K. and A.E. Shammaky, 2005. A certain subclass of meromor-phically p-valent convex functions with negative coefficients. J. Approx. Theory Appl., 1: 123-143.
Aouf, M.K. and H.M. Hossen, 1993. New criteria for meromorphic p-valent starlike functions. Tsukuba $\mathrm{J}$. Math., 1: 481-486.

Aouf, M.K. and R.M. El-Ashwah, 2009. Subclasses of meromorphically $\mathrm{p}$-valent functions with negative coefficients associated with a linear operator. Bull. Institute Math., 4: 97-118.

Aouf, M.K., 2009. Properties of certain subclasses of meromorphic functions with positive coefficients. Math. Comput. Modell., 49: 868-879.

DOI: $10.1016 /$ j.mcm.2008.04.013

Aouf, M.K., 2010. On certain subclass of meromorphic p-valent functions with negative coefficients. General Math., 18: 71-84.

Duren, P.L., 1983. Univalent Functions. 1st Edn., Springer Science and Business Media, New York, ISBN-10: 0387907955, pp: 382.

Goodman, A.W., 1957. Univalent functions and nonanalytic curves. Proc. Am. Math. Soc., 8: 598-601.

Goodman, A.W., 1983. Univalent Functions. 1st Edn., Mariner Pub Co., Florida, ISBN-10: 093616610X.

Kamali, M., K. Suchithra, B.A. Stephen and A. Gangadharan, 2011. On certain subclass of meromorphic $p$-valent functions with negative coefficients. General Math., 19: 109-122.

Liu, J.L. and H.M. Srivastava, 2004. Subclasses of meromorphically multivalent functions associated with a certain linear operator. Math. Comput. Modell., 39: 35-44.

DOI: $10.1016 / \mathrm{S} 0895-7177(04) 90504-3$

Makinde, D.O., 2012. On a certain family of meromorphic $p$-valent functions with negative coefficients. Math. Theory Model., 2: 2224-5804.

Ruscheweyh, S., 1981. Neighborhoods of univalent functions, Proc. Am. Math. Soc., 81: 521-527.

Schild, A. and H. Silverman, 1975. Convolution of univalent functions with negative coefficients. Annales Univ. Mariae Curie, 29: 99-107.

Silverman, H., 1997. Partial sums of starlike and convex functions. J. Math. Anal. Appli., 209: 221-227. DOI: $10.1006 /$ jmaa.1997.5361

Silvia, E.M., 1985. Partial sums of convex functions of order $\alpha$. Houston J. Math., 11: 397-404.

Srivastava, H.M. and S. Owa, 1992. Current Topics in Analytic Function Theory. 1st Edn., World Scientific, Singapore, ISBN-10: 9810209320, pp: 456.

Srivastava, H.M., H.M. Hossen and M.K. Aouf, 1998. A certain subclass of meromorphically convex functions with negative coefficients. Math. J. Ibaraki Univ., 30: 33-51. DOI: 10.5036/mjiu.30.33

Uralegaddi, B.A. and M. D. Ganigi, 1987. Meromorphic convex functions with negative coefficients. J. Math. Res. Exposition, 1: 21-26. 